\title{
Perioperative Antibiotic Prophylaxis: An Educative Intervention Significantly Increases Compliance
}

\author{
Eirini Chrysovalantou Martzivanou ${ }^{\mathrm{a}, \mathrm{d}}$, Kyriakos Psarras ${ }^{\mathrm{a}}$, Panagiotis Monioudis ${ }^{\mathrm{a}}$, \\ Kyriakos Vamvakis ${ }^{\mathrm{b}}$, Christina Nikolaidou ${ }^{\mathrm{a}}$, Georgios Geropoulos ${ }^{\mathrm{c}}$, \\ Efstathios T. Pavlidis ${ }^{\mathrm{a}}$, Nikolaos Symeonidis ${ }^{\mathrm{a}}$, Theodoros E. Pavlidis ${ }^{\mathrm{a}}$
}

\begin{abstract}
Background: Implementation of guidelines in daily clinical practice is often suboptimal, mainly due to doctors' poor compliance with them. Perioperative antibiotic prophylaxis (PAP) is many times administered in patients undergoing elective surgery without proper indication or for longer time than needed. Aim of this study is to investigate the effect of a simple intervention on the compliance of the medical staff with the American Society of Health-System Pharmacists (ASHP) guidelines concerning PAP administration.
\end{abstract}

Methods: A prospective 3-month audit took place including routine surgical procedures (laparoscopic cholecystectomy, inguinal hernia repair and thyroidectomy). An intervention aiming to educate the medical staff was implemented. The intervention included the demonstration of a poster and the training of the medical staff over the guidelines. A re-audit recorded the changes in daily clinical practice.

Results: The compliance rate regarding the number of PAP doses significantly increased from $0 \%$ before the intervention to $68.8 \%$ after the intervention for hernia repair and to $53.1 \%$ for laparoscopic cholecystectomy. The adherence rate in thyroidectomies increased from $25 \%$ to $50 \%$, but without statistical significance. No significant difference was found for other parameters of PAP administration, namely the type of antibiotic used and the timing of the dose administration. Regarding secondary outcomes, hospitalization days were reduced, and cost of antibiotics was significantly decreased (P $<0.05)$.

Conclusions: A simple intervention intending to educate the medi-

Manuscript submitted February 14, 2021, accepted March 1, 2021

Published online March 19, 2021

aSecond Surgical Propedeutic Department, Hippocration Hospital, Aristotle University of Thessaloniki, Thessaloniki, Greece

birectorate of Public Health, Prefecture of Kavala, Kavala, Greece

'Thoracic Surgery Department, University College London Hospitals, NHS Foundation Trust, London, UK

${ }^{\mathrm{d}}$ Corresponding Author: Eirini Chrysovalantou Martzivanou, Second Surgical Propedeutic Department, Hippocration Hospital, Aristotle University of Thessaloniki, Thessaloniki, Greece. Email: eirinimartzi@hotmail.com

doi: https://doi.org/10.14740/jocmr4447 cal staff was successful in achieving significant improvement on the compliance rate with the PAP guidelines, highlighting the importance of promoting adherence to the already existing, well-established guidelines.

Keywords: Antibiotic prophylaxis; Surgical site infection; Intervention; Compliance

\section{Introduction}

Although new antibiotic agents are developed and tested every day, infections continue to be difficult to handle [1]. One of the reasons of this problem is overuse and misuse of antibiotics, leading to creation of resistant bacterial agents. According to World Health Organization (WHO) Report on Surveillance of Antibiotic Consumption, Greece has the second highest rate of antibiotic consumption following Turkey [2]. Cassini et al reported that Greece and Italy are the countries with the highest numbers of infections from resistant bacteria in the European Union [3]. Solution to this problem is probably not the development of stronger antibiotic agents, but the preservation of the already existing antibiotics' effectiveness [1].

Towards this direction antimicrobial stewardship programs were created in order to educate the medical staff and promote the appropriate use of antimicrobial agents [4]. One of these programs' tools is audits. An audit is the monitoring of the compliance of health care workers with specific, widely accepted guidelines [5].

Perioperative administration of antibiotics as prophylaxis for surgical infections is one of the fields that antibiotic consumption can easily be monitored and its misuse restricted.

Different studies examined the compliance of the medical staff with specific guidelines regarding PAP and proved that the rates are indeed quite low $[6,7]$. According to a WHO factsheet either the medical practitioners are not adequately informed about the guidelines or the surveillance of their implementation is unsatisfactory [8].

Our study aims to investigate whether the compliance of the medical staff can improve with an educative intervention involving easily accessible and comprehensible information 


\section{ANTIBIOTIC PROPHYLAXIS GUIDELINES}

ASHP Guidelines

- Laparoscopic

Cholecystectomy

- Inguinal hernia

repair

- Thyroidectomy
Low risk patients: no AP (Strength of evidence=A)

High risk patients (for example diabetes, anticipated procedure duration exceeding 120 minutes, risk of intraoperative gallbladder rupture, age of $>70 y e a r s$, acute cholecystitis, anticipated bile spillage, jaundice, nonfunctioning gallbladder, and immunosuppression): a single dose of AP Guidelines suggest a single dose of antimicrobial prophylaxis to all patients undergoing laparoscopic cholecystectomy.
A single dose of second-generation cephalosporin (cefoxitin).

For patients with MRSA, add a single preoperative dose of vancomycin. (Strength of evidence for prophylaxis $=$ A.)

\section{No AP recommended.}

With placement of prosthetic material: a preoperative dose of cefazolin or cefuroxime is recommended. (Strength of evidence against prophylaxis without prosthesis placement $=\mathrm{B}$; strength of evidence for prophylaxis with prosthesis placement $=$ C.)

Auwaerter PG, Weinstein RA, Napolitano LM, Bratzler DW, Fish DN, Bolon MK, et al. Clinical practice guidelines for antimicrobial prophylaxis in surgery. Am J Heal Pharm. 2013;70(3):195-283.

AP: Antibiotic Prophylaxis MRSA: Methicillin-resistant Staphvlococcus aureus

Figure 1. Antibiotic prophylaxis guidelines poster.

over the specific field of PAP.

\section{Materials and Methods}

A single-institution prospective audit and re-audit were con- ducted between November 2018 and April 2019, and included adults undergoing elective laparoscopic cholecystectomy, inguinal hernia repair or thyroidectomy. Following an initial audit which took place between November 2018 and January 2019 , the medical staff was trained, by means of a poster (Fig. 1) and an explanatory session about the guidelines regard- 
Table 1. Number of Patients per Operation and in Total, Before and After the Intervention

\begin{tabular}{lll} 
& Before intervention & After intervention \\
\hline Laparoscopic cholecystectomy & 37 & 32 \\
Hernia repair & 25 & 16 \\
Thyroidectomy & 4 & 2 \\
Total & 66 & 50 \\
\hline
\end{tabular}

ing PAP [9]. The medical staff included the general surgeons, the residents and the nurses of one single surgical ward. The explanatory session was a one-point intervention, where the guidelines were explained and a poster with the guidelines written very simply (Fig. 1) was displayed at the doctors' and nurses' working rooms for the duration of the re-audit.

The re-audit was conducted between February 2019 and April 2019. Informed consent by all participants in the study was obtained. The study protocol was approved by the hospital's ethics committee and received the Institutional Review Board approval. The study protocol was in accordance with The Code of Ethics of the World Medical Association (Declaration of Helsinki).

The main data collected for every patient were the timing of the administration of the antibiotic prophylaxis, the type of the antibiotic prescribed and the number of doses administered. History of anaphylactic reaction to antibiotics was also recorded. Secondary data included the days of hospitalization and the cost effect of PAP overuse. Other data collected were age, sex and diabetes status.

In order to monitor the compliance rate, a single dose of antibiotic prophylaxis (AP) was reckoned as compliance with the guidelines for elective laparoscopic cholecystectomy $[9,10]$. For inguinal hernia repair procedure (mesh-plug technique), the recommended AP is a single dose of a first-generation cephalosporin (cefazolin) (strength of evidence: A). Instead of cefazolin, the administration of a single dose of a secondgeneration cephalosporin (cefoxitin) was considered as baseline prophylaxis by the local infectious diseases committee. Thyroidectomy is considered as a clean procedure, so no AP is recommended (strength of evidence: B), except in the case of placement of a prosthetic material, such as a drain, where the administration of a single dose of cefazolin or cefuroxime preoperatively is recommended (strength of evidence: C).

Ciprofloxacin was administered to patients allergic to penicillin or cephalosporins.

The timing of PAP administration was recorded by an in- vestigator who noted whether the AP was administered "within 60 min" before surgical incision or not.

The primary outcome of the study was to estimate the adherence to the guidelines regarding the AP. Secondary outcomes were the cost of the antibiotics administered and the days of hospitalization.

The statistical analysis was performed by using the R studio (version 1.1.442 ${ }^{\odot} 2009$ - 2018 RStudio, Inc.). The study population characteristics are presented as mean and standard deviation or median and interquartile range. The compliance rate was estimated by using Chi-squared test or Fisher's exact test when needed. The significance level was set to 0.05 .

\section{Results}

A total of 116 patients were enrolled in the study. Sixty-six patients took part at the initial audit and 50 patients at the post-intervention re-audit (Table 1). Patient characteristics are shown in Table 2. Age, sex, diabetes mellitus status and history of allergy demonstrate no statistically significant differences between the study population before and after the intervention.

Concerning the number of PAP doses, it was found that before the intervention there was not adherence to the guidelines for hernia repair and laparoscopic cholecystectomy with a mean number of four PAP doses administered. After the intervention, compliance improved significantly to $68.8 \%$ ( $\mathrm{P}<$ $0.05)$ for hernia repair and 53.1\% $(\mathrm{P}<0.05)$ for laparoscopic cholecystectomy. Compliance in thyroidectomies before and after the intervention was $25 \%$ and $50 \%$ respectively without significant difference $(\mathrm{P}>0.05)$. The overall compliance regarding the number of PAP doses increased from $1.5 \%$ to $58 \%$ $(\mathrm{P}<0.05)$ (Table 3).

Cefoxitin was used in almost all the patients, except in the case of history of allergy when ciprofloxacin was used instead. In some cases, intravenous cefoxitin was converted to cefuroxime per os. Intraoperative bile spillage because of rupture

Table 2. Patients' Characteristics Before and After the Intervention

\begin{tabular}{llll}
\hline & Before intervention & After intervention & P value \\
\hline Age (years), mean & $58.15 \pm 14.05$ & $53.82 \pm 17.82$ & 0.1598 \\
Sex & & & \\
\multicolumn{1}{r}{ Male } & $39 / 66$ & $30 / 50$ & 0.9213 \\
$\quad$ Female & $27 / 66$ & $20 / 50$ & 0.2605 \\
Diabetes mellitus & $7.6 \%$ & $14 \%$ & 0.1388 \\
Allergy history & $9.1 \%$ & $2 \%$ & \\
\hline
\end{tabular}


Table 3. Compliance With the Dose of Perioperative Antibiotic Prophylaxis per Operation and in Total, Before and After The Intervention

\begin{tabular}{lllll} 
& ASHP criteria & Before intervention & After intervention & P value \\
Hernia repair & One single dose, preoperatively & $0 \%$ & $68.8 \%$ & P $<0.005$ \\
Thyroidectomy & AP depending on prosthetic material & $25 \%$ & $50 \%$ & 1 \\
Laparoscopic cholecystectomy & One single dose, preoperatively & $0 \%$ & $53.1 \%$ & $\mathrm{P}<0.005$ \\
All operations & & $1.5 \%$ & $58 \%$ & $\mathrm{P}<0.005$ \\
\hline
\end{tabular}

ASHP: American Society of Health-System Pharmacists; AP: antibiotic prophylaxis.

Table 4. Number of Doses of Antibiotics Before and After the Educational Intervention

\begin{tabular}{lll} 
& Before intervention & After intervention \\
\hline Cefoxitin & 269 & 132 \\
Metronidazole & 15 & 65 \\
Ciprofloxacin & 13 & 9 \\
Amikacin & 3 & - \\
Cefuroxime & - & 32 \\
\hline
\end{tabular}

of the gallbladder due to manipulations led surgeons to add metronidazole alongside the standard antibiotic used as PAP $(1.5 \%$ of cases before the intervention and $12 \%$ of cases after the intervention). In one case before the intervention amikacin was administered along with cefoxitin and metronidazole (Table 4).

According to the American Society of Health-System Pharmacists (ASHP) guidelines, the antibiotic prophylaxis should be administered "within 60 min" before surgical incision. The results showed no statistically significant difference regarding this parameter before and after the intervention. The compliance rates for all the three types of operations ranged around $50 \%(\mathrm{P}>0.05$, Table 5).

Secondary aims were the cost of the PAP and the days of hospitalization before and after the intervention.

We estimated that the cost of the antibiotics decreased significantly $(\mathrm{P}<0.05)$. The cost decreased from a median of
$€ 15.08$ before the intervention to a median of $€ 3.77$ after the intervention (Table 6).

Days of hospitalization were statistically decreased $(\mathrm{P}=$ 0.046). Patients before the intervention stayed in hospital a median of 6 days and after the intervention a median of 4 days (Table 7).

\section{Discussion}

The aim of this study was to examine how a simple intervention could affect the standard routine of a single surgical department. The observation that some surgical patients undergoing the same operation were not receiving the same PAP, led to the finding that this approach was not in accordance with the recent PAP guidelines. The audit and the re-audit that took place demonstrated that single actions such as inform-

Table 5. Compliance With the Timing of Administration per Operation and in Total, Before and After the Educational Intervention

\begin{tabular}{llll}
\hline & Before intervention & After intervention & P value \\
\hline Hernia repair & $57.14 \%$ & $42.86 \%$ & 0.61 \\
Thyroidectomy & $50 \%$ & $50 \%$ & 0.47 \\
Laparoscopic cholecystectomy & $50.88 \%$ & $49.12 \%$ & 0.32 \\
All operations & $52.44 \%$ & $47.56 \%$ & 0.13 \\
\hline
\end{tabular}

Table 6. Median of Cost $(€)$ per Operation and in Total, Before and After the Educational Intervention

\begin{tabular}{llll} 
& Before intervention & After intervention & P value \\
\hline Hernia repair & 15.08 & 3.77 & $<0.001$ \\
Thyroidectomy & 13.2 & 14.72 & 0.933 \\
Laparoscopic cholecystectomy & 15.08 & 3.77 & 0.0017 \\
All operations & 15.08 & 3.77 & $<0.01$ \\
\hline
\end{tabular}


Table 7. Mean of Hospitalization Days per Operation and in Total, Before and After the Educational Intervention

\begin{tabular}{llll} 
& Before intervention & After intervention & P value \\
\hline Hernia repair & 5.2 & 4.81 & 0.291 \\
Thyroidectomy & 7.25 & 4 & 0.4 \\
Laparoscopic cholecystectomy & 5.11 & 4.77 & 0.304 \\
All operations & 6 & 4 & 0.046 \\
\hline
\end{tabular}

ing the medical staff and providing them with written material, namely an easily readable poster, were enough to significantly improve compliance with the guidelines.

Concerning the strengths, this study was innovative, because clinicians in Greece are not familiar with the concept of audits and interventions. People that were involved, had access to the guidelines all the time and they were written in a very comprehensible way. The intervention was plain, economical and easily reproducible.

Patients' characteristics did not differ between the pre-intervention and the post-intervention group, so surgeons' choice of PAP administration was not influenced by patients' parameters. Our findings indicate that lack of knowledge is the main reason of the pre-intervention low adherence rate, since a simple notification greatly improved the situation. One study conducted in UK managed to prove such an association between the intervention and the audits results, by using a questionnaire that examined the medical staff's knowledge of PAP guidelines before and after an educative intervention [11].

Guidelines adherence varies significantly between different studies. Van Kasteren et al describe a low rate of overall compliance (28\% full adherence) while the adherence to the separate parameters of PAP is quite high [6]. Hohmann et al, on the contrary, included pharmacy interns in the study and estimated a $70.7 \%$ overall compliance to the guidelines [12]. Palacios-Saucedo et al implemented an intervention that included both hard and electronic copies of the guidelines and notification of every doctor over their individual compliance rate. Results showed significant improvement $(\mathrm{P}<0.05)$ to all parameters of PAP [13]. In a similar study by Brink et al, face to face sessions were used as quality improvement techniques; and a $24.7 \%(\mathrm{P}<0.0001)$ increase in overall compliance with PAP guidelines was found [14]. Many published data describe similar intervention with similar results $[15,16]$.

In opposition to the present study, Knox et al report a 3\% decrease $(\mathrm{P}>0.05)$ in overall compliance after the application of an intervention, including display of the guidelines at the department's ward and on the hospital's site [17]. A decrease of about $6 \%(\mathrm{P}=0.59)$ in total adherence rate is described by Ozgun et al despite the informative sessions over prudent PAP administration [18]. Similar results from other studies $[19,20]$ prove that interventions do not always return positive outcomes.

One of the limitations of the study is that anesthesiologists were not involved in the intervention. This fact can explain why the compliance with the timing of administration showed no statistically significant difference, since surgeons are responsible for the type of antibiotic that will be used as PAP, but anesthesiologists are responsible for the timing of the AP administration in the operating theater.

Another limitation of the study is that we did not take into consideration the weight of the patients. According to the ASHP guidelines, the dose of the antibiotic should be weightadjusted. Both before and after the intervention, a standard dose of PAP was administered in the study regardless of the patients' weight. This parameter was neither included in our poster nor explained to the medical staff.

The study proved that a simple intervention was enough to cause a significant improvement, regarding the appropriate dosing of PAP according to the ASHP guidelines, for a short period of time. The long-term results of the study may differ from the short-term results, since the intervention was a one-point interference and not a continuous feedback with the ability to detect and correct emerging mistakes. The implementation of repeated interventions may be necessary in order to achieve the same rates in the long term. The long-term preservation of the compliance is very important, because in order to prevent the development of antibiotic resistance it is vital to be consistent with the prudent use of antibiotics.

Another point of attention is that the baseline compliance of our study was very low, especially for laparoscopic cholecystectomy and hernia repair. It is questionable whether the same intervention could provoke such a great improvement in compliance rates if it was applied in a department where the baseline adherence was higher. Probably, for such a case, more complicated interventions would be needed. The use of hard copies, however, distributed to the medical staff and displayed to different rooms, is considered to be an effective and time saving education method, since the medical staff has continuous access to the guidelines. The combination of this method with an educative lecture constitutes a very simple but very effective way to inform the staff for the existing guidelines.

In a similar way, cost was significantly reduced, because the compliance rate before the intervention was very low. So, it is not guaranteed that the application of the same study in another department will yield the same results.

\section{Conclusions}

Every department and every clinical practitioner individually have to make an effort to comply with the guidelines regarding the antibiotic administration. This study shows that a really simple and cost-effective intervention efficiently improved the compliance rate and subsequently promoted the prudent use of antibiotics. Further research is needed in order to design an intervention that will reduce the cost and ensure adherence to the PAP guidelines in the long term. 


\section{Acknowledgments}

The authors would thank the general surgeons in the Second Propedeutical Department of General Hospital of Thessaloniki "Ippokrateio" for their willingness to participate in the study.

\section{Financial Disclosure}

This research did not receive any specific grant from funding agencies in the public, commercial, or not-for-profit sectors.

\section{Conflict of Interest}

None to declare.

\section{Informed Consent}

Informed consent was obtained.

\section{Author Contributions}

EM and KP contributed to the conception and design of the study. EM, PM, CN and GG participated in the acquisition of data and analysis. EM, KV, EP and NS took part in the preparation of the manuscript, and TP participated in the creation of the final form of the paper. All authors approve the submitted version.

\section{Data Availability}

The data supporting the findings of this study are available from the corresponding author upon reasonable request.

\section{References}

1. Medina E, Pieper DH. Tackling threats and future problems of multidrug-resistant bacteria. Curr Top Microbiol Immunol. 2016;398:3-33.

2. World Health Organization. WHO Report on Surveillance of antibiotic consumption: 2016-2018 early implementation. Geneva; 2018.

3. Cassini A, Hogberg LD, Plachouras D, Quattrocchi A, Hoxha A, Simonsen GS, Colomb-Cotinat M, et al. Attributable deaths and disability-adjusted life-years caused by infections with antibiotic-resistant bacteria in the EU and the European Economic Area in 2015: a population-level modelling analysis. Lancet Infect Dis. 2019;19(1):56-66.

4. Pollack LA, Plachouras D, Gruhler H, Sinkowitz-cochran R. Transatlantic Taskforce on Antimicrobial Resistance (TATFAR) Summary the modified Delphi process for common structure and process indicators for hospital an- timicrobial stewardship programs. 2015.

5. Jones B, Vaux E, Olsson-Brown A. How to get started in quality improvement. BMJ. 2019;364:k5408.

6. van Kasteren ME, Kullberg BJ, de Boer AS, Mintjes-de Groot J, Gyssens IC. Adherence to local hospital guidelines for surgical antimicrobial prophylaxis: a multicentre audit in Dutch hospitals. J Antimicrob Chemother. 2003;51(6):1389-1396.

7. Mondelo Garcia C, Gutierrez Urbon JM, Perez Sanz C, Martin Herranz MI. Auditing and improving surgical antibiotic prophylaxis. Surg Infect (Larchmt). 2018;19(7):679683.

8. World Health Organization. Regulate and promote rational use of medicines, including in animal husbandry, and ensure proper patient care. 2011.

9. Bratzler DW, Dellinger EP, Olsen KM, Perl TM, Auwaerter PG, Bolon MK, Fish DN, et al. Clinical practice guidelines for antimicrobial prophylaxis in surgery. Am J Health Syst Pharm. 2013;70(3):195-283.

10. Matsui Y, Satoi S, Hirooka S, Kosaka H, Kawaura T, Kitawaki T. Reappraisal of previously reported meta-analyses on antibiotic prophylaxis for low-risk laparoscopic cholecystectomy: an overview of systematic reviews. BMJ Open. 2018;8(3):e016666.

11. Cameron M, Jones S, Adedeji O. Antibiotic prophylaxis audit and questionnaire study: Traffic Light Poster improves adherence to protocol in gastrointestinal surgery. Int J Surg. 2015;19:112-115.

12. Hohmann C, Eickhoff C, Radziwill R, Schulz M. Adherence to guidelines for antibiotic prophylaxis in surgery patients in German hospitals: a multicentre evaluation involving pharmacy interns. Infection. 2012;40(2):131-137.

13. Palacios-Saucedo GDC, de la Garza-Camargo M, Briones-Lara E, Carmona-Gonzalez S, Garcia-Cabello R, Islas-Esparza LA, Saldana-Flores G, et al. [Assessment of antibiotic use and impact of an intervention intended to modify the prescribing behavior in surgical prophylaxis in 6hospitals in the metropolitan area of Monterrey, Mexico]. Cir Cir. 2017;85(6):459-470.

14. Brink AJ, Messina AP, Feldman C, Richards GA, van den Bergh D, Netcare Antimicrobial Stewardship Study A. From guidelines to practice: a pharmacist-driven prospective audit and feedback improvement model for perioperative antibiotic prophylaxis in 34 South African hospitals. J Antimicrob Chemother. 2017;72(4):1227-1234.

15. Young BC, Hacker MR, Dodge LE, Golen TH. Timing of antibiotic administration and infectious morbidity following cesarean delivery: incorporating policy change into workflow. Arch Gynecol Obstet. 2012;285(5):12191224.

16. Sutherland T, Beloff J, Lightowler M, Liu X, Nascimben L, Kaye AD, Urman RD. Description of a multidisciplinary initiative to improve SCIP measures related to preoperative antibiotic prophylaxis compliance: a singlecenter success story. Patient Saf Surg. 2014;8(1):37.

17. Knox MC, Edye M. Educational antimicrobial stewardship intervention ineffective in changing surgical prophylactic antibiotic prescribing. Surg Infect (Larchmt). 2016;17(2):224-228. 
18. Ozgun H, Ertugrul BM, Soyder A, Ozturk B, Aydemir M. Peri-operative antibiotic prophylaxis: adherence to guidelines and effects of educational intervention. Int J Surg. 2010;8(2):159-163.

19. Korableva AA, Yudina EV, Ziganshina LE. [Efficacy of management for rational use of antibiotics in surgical departments at a multi-disciplinary hospital: results of a 7-year pharmacoepidemiological research]. Vestn Ross Akad Med Nauk. 2017;72(1):26-32.

20. Kritchevsky SB, Braun BI, Bush AJ, Bozikis MR, Kusek L, Burke JP, Wong ES, et al. The effect of a quality improvement collaborative to improve antimicrobial prophylaxis in surgical patients: a randomized trial. Ann Intern Med. 2008;149(7):472-480, W489-493. 